\title{
Controllability of Second-Order Multiagent Systems with Multiple Leaders and General Dynamics
}

\author{
Bo Liu, ${ }^{1}$ Hongke Feng, ${ }^{1}$ Li Wang, ${ }^{2}$ Rong Li, ${ }^{3}$ Junyan Yu, ${ }^{4}$ \\ Housheng Su, ${ }^{5}$ and Guangming Xie ${ }^{6}$ \\ ${ }^{1}$ College of Science, North China University of Technology, Beijing 100144, China \\ ${ }^{2}$ Beijing Key Lab of Urban Intelligent Traffic Control Technology, North China University of Technology, Beijing 100144, China \\ ${ }^{3}$ Department of Applied Mathematical Sciences, Shanghai Finance University, Shanghai 201209, China \\ ${ }^{4}$ College of Mathematical Science, University of Electronic Science and Technology of China, Chengdu 611731, China \\ ${ }^{5}$ Department of Control Science and Engineering, Key Laboratory of Image Processing and Intelligent Control of Education Ministry \\ of China, Huazhong University of Science and Technology, Wuhan 430074, China \\ ${ }^{6}$ Intelligent Control Laboratory, Center for Systems and Control, Department of Industrial Engineering and Management, \\ College of Engineering, Peking University, Beijing 100871, China
}

Correspondence should be addressed to Bo Liu; liuboer@163.com

Received 3 April 2013; Accepted 3 June 2013

Academic Editor: Ming Cao

Copyright (C) 2013 Bo Liu et al. This is an open access article distributed under the Creative Commons Attribution License, which permits unrestricted use, distribution, and reproduction in any medium, provided the original work is properly cited.

\begin{abstract}
This paper proposes a new second-order discrete-time multiagent model and addresses the controllability of second-order multiagent system with multiple leaders and general dynamics. The leaders play an important role in governing the other member agents to achieve any desired configuration. Some sufficient and necessary conditions are given for the controllability of the secondorder multiagent system. Moreover, the speed controllability of the second-order multiagent system with general dynamics is discussed. Particularly, it is shown that the controllability of the whole system relies on the number of leaders and the connectivity between the leaders and the members. Numerical examples illustrate the theoretical results.
\end{abstract}

\section{Introduction}

Controllability is one of the fundamental issues for coordinated control of multiagent systems which is partly due to the wide applications in communication and computation, as well as cooperative control [1-19].

So far, the issue of controllability shows new features and difficulties, and is still lacking in studies. In [4], the issue of controllability was firstly investigated by the nearest neighbor rules. Tanner had obtained necessary and sufficient conditions of the controllability for first-order multiagent dynamic systems regarding an agent as a leader or the external input. In $[5,7]$, the controllability for multiagent systems was investigated by the graph theoretic characterization. Moreover, Ji et al. [17] analyzed the multiagent controllability using tree topology. Jafari et al. [18] studied the structural controllability of multiagent systems. In $[9,15$,
16], the authors discussed the controllability of discrete-time multiagent systems with a single leader or multiple leaders on fixed networks and switching networks, respectively, and obtained the necessary or sufficient controllable conditions for multiagent systems. References $[10,14]$ studied the controllability of continuous-time multiagent systems with timedelay and switching topology, respectively.

However, most of the recent research work focuses on the controllability of single integrator or first-order multiagent dynamic systems, such as [4-19]. But the controllability of double integrator or second-order multiagent dynamic systems was seldom studied. Motivated by the works above, in this paper, we focus on discussing the controllability of second-order discrete-time multiagent systems with general dynamic topology. Some sufficient and necessary conditions for controllability are presented. The main contributions of our paper lie in the following. (1) A novel model of 
discrete-time multiagent system is a second-order. (2) The influence of leaders on the followers is investigated. (3) The controllability of such second-order discrete-time system with multiple leaders and general dynamics is considered, which cannot be found in the recent literatures. (4) The controllability and the speed controllability of the secondorder multiagent system are discussed, respectively. (5) A sufficient and necessary condition for controllability of the second-order system is presented.

This paper is organized as follows. In Section 2, we present some concepts in graph theory. Section 3 gives the model to be studied. In Section 4, main results are presented. In Section 5, numerical examples and simulations are provided to illustrate the theoretical results. A conclusion is made in Section 6.

\section{Preliminaries}

In this section, some basic definitions and concepts in graph theory [20] are first introduced.

Let $\mathscr{G}=(\mathscr{V}, \mathscr{E}, A)$ be an undirected graph of order $n$ with the set of nodes $\mathscr{V}=\{1,2,3, \ldots, n\}$ and the set of edges $\mathscr{E} \subseteq \mathscr{V} \times \mathscr{V}$. An edge of $\mathscr{G}$ is denoted by $(i, j)$, which is an unordered pair of distinct nodes of $\mathscr{V}$. If $i, j \in \mathscr{V}$, and $(i, j) \in \mathscr{E}$, then we say that $j$ is a neighbor of $i$ or $i$ and $j$ are adjacent. The neighborhood set of node $i$ is denoted by $\mathcal{N}_{i}=\{j \in \mathscr{V}:(i, j) \in \mathscr{E}\} . A=\left[a_{i j}\right] \in \mathbb{R}^{n \times n}$, where $a_{i j}>0 \Leftrightarrow(j, i) \in \mathscr{E}$, and $a_{i j}$ is called the coupling weight of edge $(i, j)$.

Any undirected graph can be represented by its adjacency matrix $A$, which is a symmetric matrix. A diagonal matrix $D=\operatorname{diag}\left\{d_{1}, d_{2}, \ldots, d_{n}\right\} \in R^{n \times n}$ is a degree matrix of $\mathscr{G}$ with its diagonal elements $d_{i}=\sum_{j \in \mathcal{N}_{i}} a_{i j}, i=1,2, \ldots, n$. fined as

Then, the Laplacian of the graph $\mathscr{G}$ (or matrix $A$ ) is de-

$$
L=D-A \in R^{n \times n}
$$

\section{Model}

Consider a second-order multiagent system with $N+n_{l}$ agents, labeled the first $N$ agents from 1 to $N$ as followers and the remainder $n_{l}$ agents from $N+1$ to $N+n_{l}$ as leaders, and each agent moves according to the following dynamics:

$$
\begin{aligned}
& x_{i}(k+1)=x_{i}(k)+v_{i}(k), \\
& v_{i}(k+1)=v_{i}(k)+u_{i}(k),
\end{aligned}
$$

with

$$
\begin{aligned}
u_{i}(k)= & -\sum_{i \in \mathscr{N}_{i_{j}}} a_{i j}\left(v_{i}(k)-v_{j}(k)\right) \\
& -\sum_{p \in \mathcal{N}_{i_{p}}} \gamma_{i p} b_{i p}\left(v_{i}(k)-v_{p}(k)\right), \quad k \in J_{k},
\end{aligned}
$$

where $x_{i} \in \mathbb{R}^{m}$ is the state of agent $i(i \in \underline{N} \triangleq\{N+1, \ldots, N+$ $\left.\left.n_{l}\right\}\right)$ and $x_{p} \in \mathbb{R}^{m}$ is the state of agent $p\left(p \in N+n_{l}-\right.$ $\left.\underline{N}, N+n_{l} \triangleq\{1,2, \ldots, N\}\right) . \mathcal{N}_{i}$ presents the neighbor set of agent $i . \mathcal{N}_{i_{j}} \cup \mathcal{N}_{i_{p}}=\mathcal{N}_{i}$, and $\mathscr{N}_{i_{j}} \cap \mathcal{N}_{i_{p}}=\emptyset . J_{k}$ is a discretetime index set. The coupling matrix $A=\left[a_{i j}\right] \in \mathbb{R}^{N \times N}$ with $a_{i j} \geq 0$ and $a_{i i}=0$ represents the coupling strength among the followers, and $B=\left[b_{i p}\right] \in \mathbb{R}^{N \times n_{l}}$ with $b_{i p}>0$ represents the coupling strength from the leaders to the followers. $\gamma_{i p}=1$ if there is information from leader $p$ to follower $i$; otherwise $\gamma_{i p}=0$.

Throughout this paper, it is assumed that the leader can influence the member followers but cannot be influenced by its neighbors.

Suppose $z=\left(x_{1}, x_{2}, \ldots, x_{N}, v_{1}, v_{2}, \ldots, x_{N}\right)^{T}$ and $y=$ $\left(x_{N+1}, x_{N+2}, \ldots, x_{N+n_{l}}, v_{N+1}, v_{N+2}, \ldots, v_{N+n_{l}}\right)^{T}$ be the state vector of all the followers and all the leaders, respectively. Then, (2) can be rewritten as

$$
z(k+1)=G z(k)+H y(k), \quad k \in J_{k},
$$

where

$$
G=\left(\begin{array}{cc}
I & I \\
0 & F
\end{array}\right), \quad H=\left(\begin{array}{ll}
0 & 0 \\
0 & P
\end{array}\right),
$$

$F=I-L-R, I$ is the $N \times N$ identity matrix,

$$
\begin{gathered}
R=\operatorname{diag}\left\{\sum_{p} \gamma_{1 p} b_{1 p}, \sum_{p} \gamma_{2 p} b_{2 p}, \ldots, \sum_{p} \gamma_{N p} b_{N p}\right\} \in \mathbb{R}^{N \times N}, \\
P=\left[\begin{array}{cccc}
\gamma_{1(N+1)} b_{1(N+1)} & \gamma_{1(N+2)} b_{1(N+2)} & \cdots & \gamma_{1\left(N+n_{l}\right)} b_{1\left(N+n_{l}\right)} \\
\gamma_{2(N+1)} b_{2(N+1)} & \gamma_{2(N+2)} b_{2(N+2)} & \cdots & \gamma_{2\left(N+n_{l}\right)} b_{2\left(N+n_{l}\right)} \\
\vdots & \vdots & \ddots & \vdots \\
\gamma_{N(N+1)} b_{N(N+1)} & \gamma_{N(N+2)} b_{N(N+2)} & \cdots & \gamma_{N\left(N+n_{l}\right)} b_{N\left(N+n_{l}\right)}
\end{array}\right] .
\end{gathered}
$$

For simplicity, we denote (6) as

$$
P=\left[p_{N+1}, p_{N+2}, \ldots, p_{N+n_{l}}\right] \in \mathbb{R}^{N \times n_{l}},
$$

where

$$
\begin{array}{r}
p_{p}=\left(\gamma_{1 p} b_{1 p}, \gamma_{2 p} b_{2 p}, \ldots, \gamma_{N p} b_{N p}\right)^{T} \in \mathbb{R}^{N \times 1}, \\
q \in \underline{N+n_{l}}-\underline{N},
\end{array}
$$

and the matrix $L=\left[l_{i j}\right]$ with

$$
l_{i j}= \begin{cases}-a_{i j}, & i \neq j \text { and } j \in \mathcal{N}_{i_{j}} \\ \sum_{j \in \mathcal{N}_{i_{j}}} a_{i j}, & i=j \\ 0, & \text { otherwise. }\end{cases}
$$

It can be easily seen that the matrix $L$ satisfies the following:

(i) the off-diagonal elements are all negative or zero;

(ii) the row sums are equal to the column sums with the value of zero. 


\section{Main Results}

In the following, we first give the definition of controllability in second-order discrete-time system and the classical criterion of controllability.

Definition 1. A nonzero state $z_{0}$ of system (4) is controllable at the initial time $h \in J_{k}$ if there exists a finite time $l \in J_{k}, l>h$ and a control input $y(k)$, such that $z(h)=z_{0}$ and $z(l)=0$. If any nonzero state $z_{0}$ of system (4) is controllable, then system (4) is said to be controllable. If $x(h)=x_{0}$ and $x(l)=0$, then system (4) is position controllable, and if $v(h)=v_{0}$ and $v(l)=$ 0 , then system (4) is speed controllable.

Definition 2 (controllability matrix). The controllability matrix of system (4) is given by

$$
Q=\left[\begin{array}{lllll}
H & G H & G^{2} H & \cdots & G^{2 N-1} H
\end{array}\right],
$$

where matrix $Q \in \mathbb{R}^{2 N \times N n_{l}}$.

Lemma 3 (Rank test for controllability). System (4) is controllable if $\operatorname{Rank}(Q)=2 N$.

Lemma 4 (PBH rank test for discrete-time systems). System (4) is controllable if (4) satisfies one of the following conditions:

(i) $\operatorname{rank}(s I-G, H)=2 N$, for all $s \in \mathbb{C}$;

(ii) $\operatorname{rank}\left(\lambda_{i} I-G, H\right)=2 N$, where $\lambda_{i}$, for all $i=1,2, \ldots$, $2 N$, is the eigenvalue of matrix $G$.

In general, for second-order multiagent systems, the controllable matrix is too hard to calculate. Therefore, we can use the $\mathrm{PBH}$ rank rest to justify the controllability of such system. In the following, we will give a more simple and convenient theorem using the $\mathrm{PBH}$ rank rest.

Theorem 5. System (4) is controllable if $\operatorname{Rank}(P)=m$.

Proof. By Lemma 4, system (4) is controllability if $\operatorname{rank}\left(\lambda_{i} I-\right.$ $G, H)=2 N$, where $\lambda_{i}$, for all $i=1,2, \ldots, 2 N$, is the eigenvalue of matrix $G$. Then, it is obvious to see that

$$
\operatorname{rank}\left(\lambda_{i} \mathbf{I}-G \quad H\right)=\operatorname{rank}\left(\begin{array}{ccc}
\lambda_{i} I-I & -I & 0 \\
0 & \lambda_{i} I-F & P
\end{array}\right)=2 N,
$$

if $\operatorname{Rank}(P)=m$.

Remark 6. From Theorem 5, we can find that the secondorder multiagent system (4) is controllable if $n_{l} \geq N\left(n_{l}\right.$ is the number of leaders and $N$ the number of followers); otherwise, the system is always uncontrollable.

Remark 7. Notice that the direct consequence of Theorem 5 is that the controllability of the network (4) of a group of agents relies only on the connectivity between the leaders and members, regardless of the connectivity of the members in the network.

Corollary 8. If Rank $(P)=N$, system (4) is speed controllable.

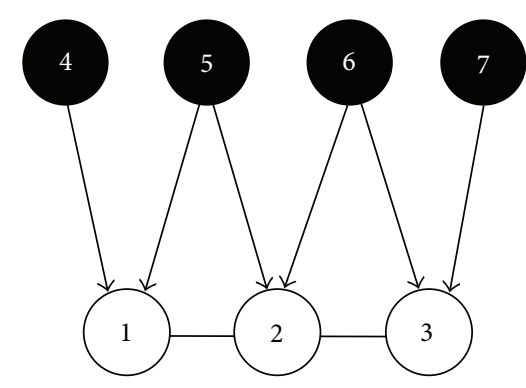

FIGURE 1: The network topology 1.

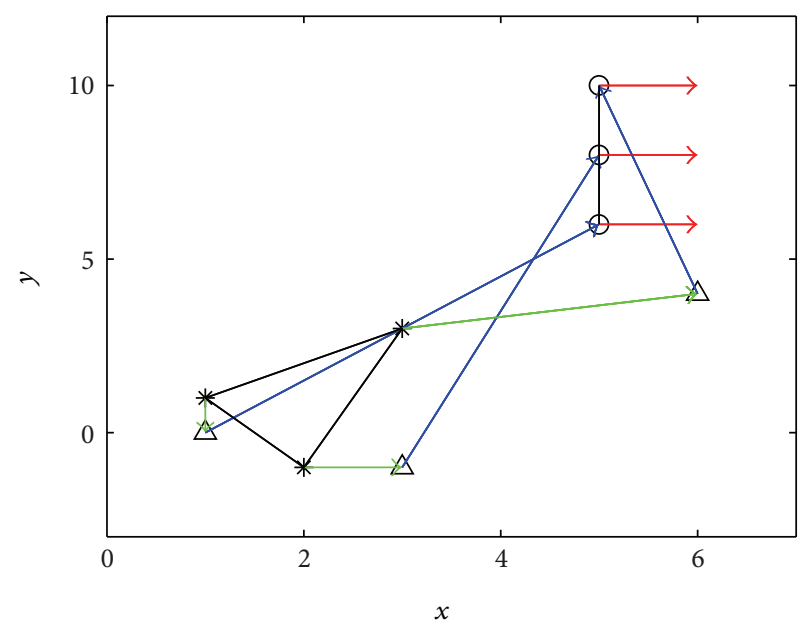

FIGURE 2: A straight line configuration with speed.

Under the symmetry condition of the adjacent matrix $A$, we can have the following result.

Theorem 9. System (4) is speed controllable if and only if the following conditions hold.

(i) The eigenvalues of $F$ are all distinct.

(ii) All the eigenvectors of $F$ are not orthogonal to at least one column in P simultaneously.

The proof of Lemma 3 is similar to that of [15, Theorem 1], here omitted.

Remark 10. From Corollary 8 and Theorem 9, it can be easily seen that even though system (4) is speed controllable, system (4) cannot be completely controllable.

\section{Numerical Examples and Simulations}

This section presents some numerical examples and simulations to illustrate the theoretical results.

Example 1. Consider a seven-agent network with agents 4-7 as the leaders, where the topology of the network is described by Figure 1. From Figure 1, we can see that the number of the leaders is more than that of the followers. For simplicity, let 


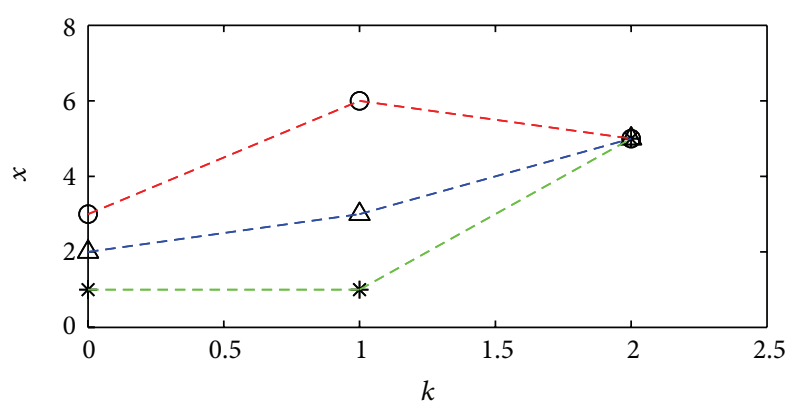

(a) Position ( $x$-axis)

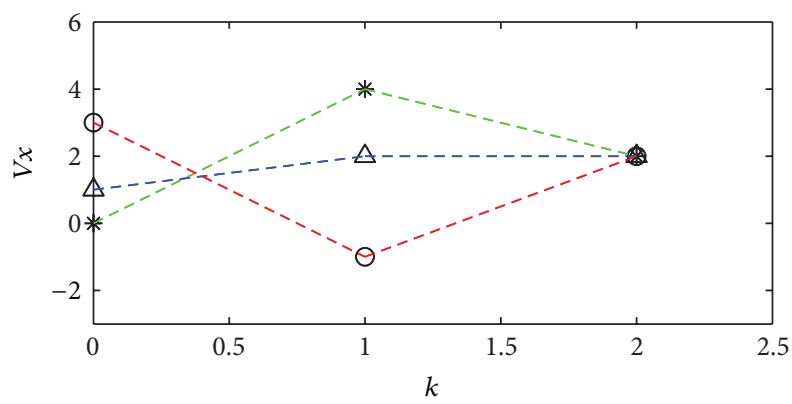

(c) Velocity ( $x$-axis)

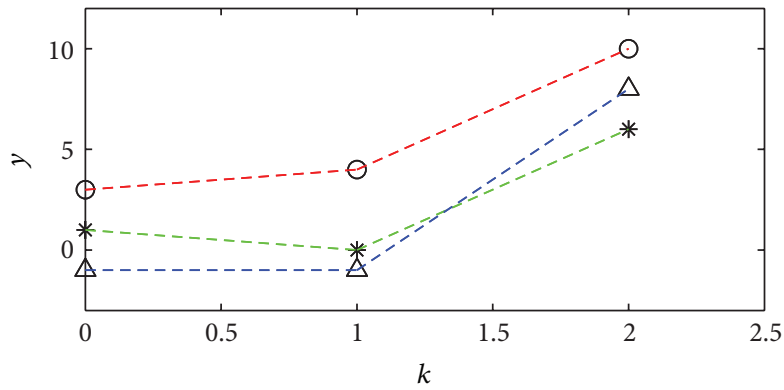

(b) Position ( $y$-axis)

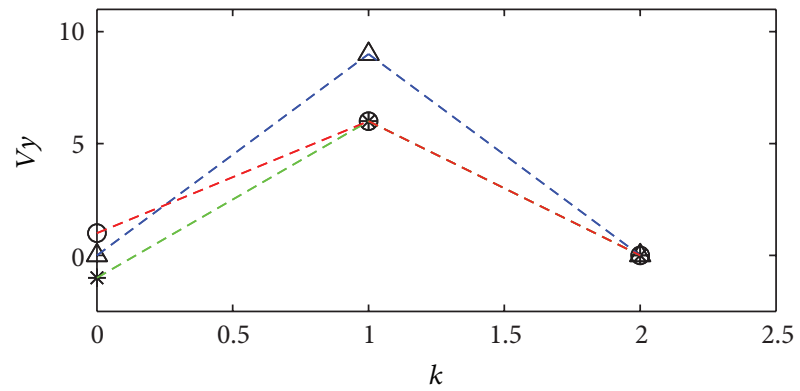

(d) Velocity ( $y$-axis)

Figure 3: Second-order controllability.

$a_{i j}=b_{i p}=1$ for $i, j=1,2,3$ and $p=4,5,6,7$. According to Figure 1, the second-order multiagent system (4) is defined by

$$
G=\left[\begin{array}{cccccc}
1 & 0 & 0 & 1 & 0 & 0 \\
0 & 1 & 0 & 0 & 1 & 0 \\
0 & 0 & 1 & 0 & 0 & 1 \\
0 & 0 & 0 & -2 & 1 & 0 \\
0 & 0 & 0 & 1 & -3 & 1 \\
0 & 0 & 0 & 0 & 1 & -2
\end{array}\right], \quad H=\left[\begin{array}{cccc}
0 & 0 & 0 & 0 \\
0 & 0 & 0 & 0 \\
0 & 0 & 0 & 0 \\
1 & 1 & 0 & 0 \\
0 & 1 & 1 & 0 \\
0 & 0 & 1 & 1
\end{array}\right],
$$

with

$$
\begin{gathered}
L=\left[\begin{array}{ccc}
1 & -1 & 0 \\
-1 & 2 & -1 \\
0 & -1 & 1
\end{array}\right], \quad R=\left[\begin{array}{lll}
2 & 0 & 0 \\
0 & 2 & 0 \\
0 & 0 & 2
\end{array}\right], \\
P=\left[\begin{array}{llll}
1 & 1 & 0 & 0 \\
0 & 1 & 1 & 0 \\
0 & 0 & 1 & 1
\end{array}\right] .
\end{gathered}
$$

By computing, $\operatorname{Rank}(P)=3$ and $\operatorname{Rank}(Q)=$ $\operatorname{Rank}\left[\begin{array}{lllll}H & G H & G^{2} H & \cdots & G^{2 N-1} H\end{array}\right]=6$, then system (4) is indeed controllable.

Figures 2 and 3 show the simulation results of formation control of the second-order network. The follower agents (the black star dots) move from a random initial configuration to desired ones: aligning in a straight line (the black circles) with controllable speed as shown in Figures 2 and 3, respectively.

Example 2. A five-agent network with agents 4 and 5 as the leaders and with fixed topology described by the graph in

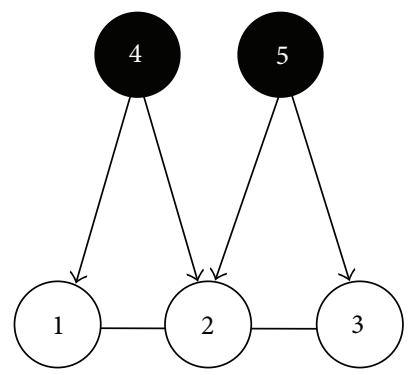

FIgURE 4: The network topology 2.

Figure 4. From Figure 4, we can see that the number of the leaders is less than that of the followers.

From Figure 1, the second-order multiagent system (4) is given by

$$
G=\left[\begin{array}{cccccc}
1 & 0 & 0 & 1 & 0 & 0 \\
0 & 1 & 0 & 0 & 1 & 0 \\
0 & 0 & 1 & 0 & 0 & 1 \\
0 & 0 & 0 & -1 & 1 & 0 \\
0 & 0 & 0 & 1 & -3 & 1 \\
0 & 0 & 0 & 0 & 1 & -1
\end{array}\right], \quad H=\left[\begin{array}{ll}
0 & 0 \\
0 & 0 \\
0 & 0 \\
1 & 0 \\
1 & 1 \\
0 & 1
\end{array}\right],
$$

with

$$
L=\left[\begin{array}{ccc}
1 & -1 & 0 \\
-1 & 2 & -1 \\
0 & -1 & 1
\end{array}\right], \quad R=\left[\begin{array}{lll}
1 & 0 & 0 \\
0 & 2 & 0 \\
0 & 0 & 1
\end{array}\right], \quad P=\left[\begin{array}{ll}
1 & 0 \\
1 & 1 \\
0 & 1
\end{array}\right]
$$




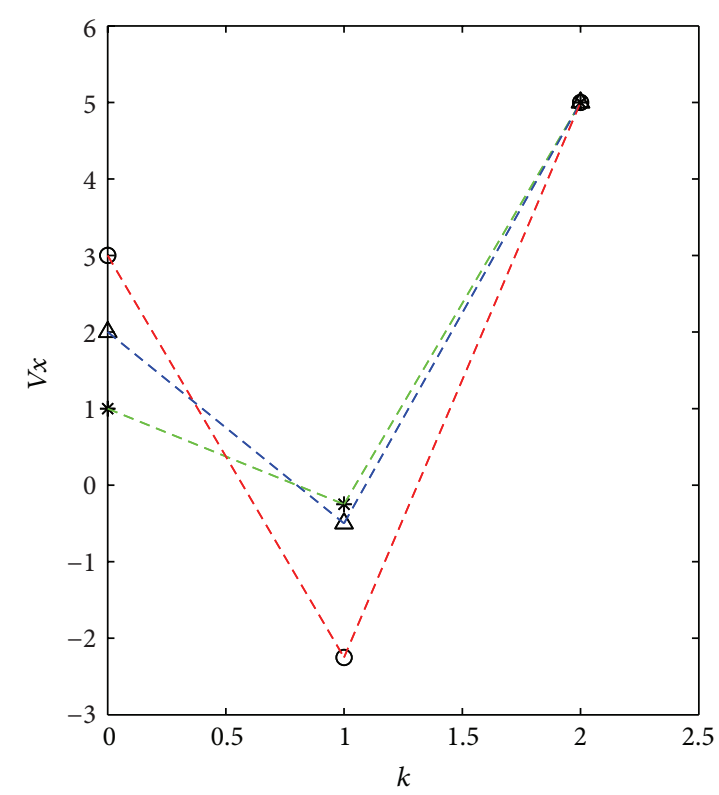

(a) Velocity ( $x$-axis)

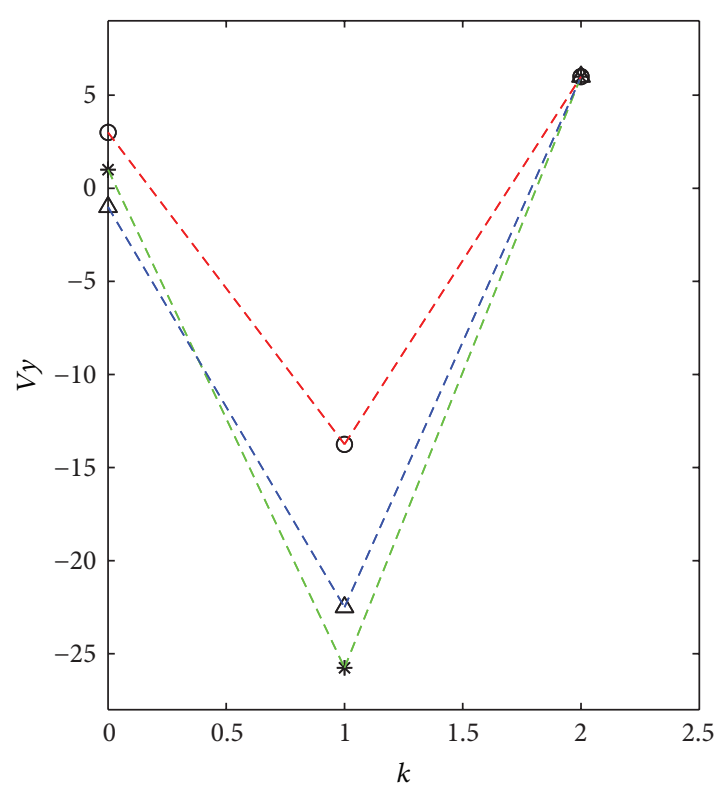

(b) Velocity ( $y$-axis)

FIGURE 5: Speed controllability.

Using Matlab calculation, the eigenvalues of $F$ are

$$
\begin{gathered}
\operatorname{eig}(F)=\{-3.7321,-1,-0.2679\}, \\
U^{T}=\left[\begin{array}{ccc}
-0.3251 & 0.8881 & -0.3251 \\
-0.7071 & -0.0000 & 0.7071 \\
0.6280 & 0.4597 & 0.6280
\end{array}\right], \\
U^{T} P=\left[\begin{array}{cc}
0.5630 & 0.5630 \\
-0.7071 & 0.7071 \\
1.0877 & 1.0877
\end{array}\right] .
\end{gathered}
$$

It is obvious that the eigenvalues of $F$ are all different, and the elements of $U^{T} P$ are all nonzero. Therefore, system (4) is speed controllable. However, $\operatorname{Rank}(P)=2$ and $\operatorname{Rank}(Q)=$ $\operatorname{Rank}\left[\begin{array}{lllll}H & G H & G^{2} H & \cdots & G^{2 N-1} H\end{array}\right]=5$, then system (4) is uncontrollable.

Figure 5 shows the simulation results of the secondorder network. It is easily seen that the speeds of the system are controllable, but the positions of the system are uncontrollable. Therefore, the whole second-order system is uncontrollable.

\section{Conclusion}

This paper has studied the controllability of discrete-time second-order multiagent systems with multiple leaders and general dynamic topology. By applying the $\mathrm{PBH}$ rank test technique, some effective sufficient and necessary conditions for the controllability of the multiagent discrete-time systems are obtained. The results in this paper show that the controllability of discrete-time second-order multiagent systems can only depend on the information from the leaders to the followers, regardless of the connectivity of the members in the network. These studies are helpful in understanding the dynamics of interconnected systems. However, for discretetime second-order multiagent systems, as in cases often encountered in practice, it is usually difficult to deal with the controllability problem due to complexity of the topology and lack of theoretical tools. Our main result shows an advantage of the second-order topology scheme. We anticipate that our solutions to the above-described problems will offer a theoretical basis and valuable ideas for future applications of networked multiagent systems in the field of coordination control, including formation control and tracking problems.

\section{Acknowledgments}

This work was supported by National Natural Science Foundation (61170113, 61203150, 61104141, 60774089, 10972003, 61174116), Science and Technology Development Plan Project of Beijing Education Commission (no. KM201310009011), the Funding Project for Academic Human Resources Development in Institutions of Higher Learning Under the Jurisdiction of Beijing Municipality (PHR201108055), the Program for New Century Excellent Talents in University from Chinese Ministry of Education under Grant NCET-12-0215, the National High Technology Research and Development Program of China 863 (no. 2012AA112401), and the Research Fund for the Doctoral Program of Higher Education (RFDP) under Grant no. 20100142120023. This work was also supported by the Foundation Grant of Guangxi Key Laboratory of Automobile Components and Vehicle Technology (13-A03-01) and the Opening Project of Guangxi Key Laboratory of Automobile Components and Vehicle Technology (2012KFZD03). 


\section{References}

[1] W. Ren and R. W. Beard, "Consensus seeking in multiagent systems under dynamically changing interaction topologies," Institute of Electrical and Electronics Engineers, vol. 50, no. 5, pp. 655-661, 2005.

[2] H. Su, X. Wang, and Z. Lin, "Flocking of multi-agents with a virtual leader," Institute of Electrical and Electronics Engineers, vol. 54, no. 2, pp. 293-307, 2009.

[3] H. Su, G. Chen, X. Wang, and Z. Lin, "Adaptive secondorder consensus of networked mobile agents with nonlinear dynamics," Automatica, vol. 47, no. 2, pp. 368-375, 2011.

[4] H. G. Tanner, "On the controllability of nearest neighbor interconnections," in Proceedings of the 43rd IEEE Conference on Decision and Control (CDC '04), pp. 2467-2472, December 2004.

[5] M. Ji and M. Egerstedt, "A graph theoretic characterization of controllability for multi-agent systems," IEEE Transactions on Robotics, vol. 23, no. 4, pp. 693-703, 2007.

[6] B. Liu, G. Xie, T. Chu, and L. Wang, "Controllability of interconnected systems via switching networks with a leader," in Proceedings of the IEEE International Conference on Systems, Man and Cybernetics, vol. 5, pp. 3912-3916, October 2006.

[7] Z. Ji, H. Lin, and T. H. Lee, "A graph theory based characterization of controllability for multi-agent systems with fixed topology," in Proceedings of the 47th IEEE Conference on Decision and Control (CDC '08), pp. 5262-5267, Cancun, Mexico, December 2008.

[8] M.Zamani and H. Lin, "Structural controllability of multi-agent systems," in Proceedings of the American Control Conference (ACC '09), pp. 5743-5748, St. Louis, Mo, USA, June 2009.

[9] B. Liu, T. Chu, L. Wang, and G. Xie, "Controllability of a leaderfollower dynamic network with switching topology," Institute of Electrical and Electronics Engineers, vol. 53, no. 4, pp. 1009-1013, 2008.

[10] Z. Ji, Z. Wang, H. Lin, and Z. Wang, "Controllability of multiagent systems with time-delay in state and switching topology," International Journal of Control, vol. 83, no. 2, pp. 371-386, 2010.

[11] R. Lozano, M. W. Spong, J. A. Guerrero, and N. Chopra, "Controllability and observability of leader-based multi-agent systems," in Proceedings of the 47th IEEE Conference on Decision and Control (CDC'08), pp. 3713-3718, Cancun, Mexico, December 2008 .

[12] L. Wang, F. Jiang, G. Xie, and Z. Ji, "Controllability of multiagent systems based on agreement protocols," Science in China F, vol. 52, no. 11, pp. 2074-2088, 2009.

[13] F. Jiang, L. Wang, G. Xie, Z. Ji, and Y. Jia, "On the controllability of multiple dynamic agents with fixed topology," in Proceedings of the American Control Conference (ACC '09), pp. 5665-5670, St. Louis, Mo, USA, June 2009.

[14] B. Liu, T. Chu, L. Wang, Z. Zuo, G. Chen, and H. Su, "Controllability of switching networks of multi-agent systems," International Journal of Robust and Nonlinear Control, vol. 22, no. 6, pp. 630-644, 2012.

[15] B. Liu, N. Hu, J. Zhang, and H. Su, "Controllability of discretetime multi-agent systems with multiple leaders on fixed networks," Communications in Theoretical Physics, vol. 58, pp. 856862, 2012.

[16] B. Liu, W. Hu, and Z. Jie, "Controllability of discrete-time multiagent systems with leaders via switching topology," Applied Mechanics and Materials, vol. 128-129, pp. 92-95, 2012.
[17] Z. Ji, H. Lin, T. H. Lee, and Q. Ling, "Multi-agent controllability with tree topology," in Proceedings of the American Control Conference (ACC '10), pp. 850-855, Baltimore, Md, USA, July 2010.

[18] S. Jafari, A. Ajorlou, A. G. Aghdam, and S. Tafazoli, "On the structural controllability of multi-agent systems subject to failure: a graph-theoretic approach," in Proceedings of the 49th IEEE Conference on Decision and Control (CDC '10), pp. 45654570, Atlanta, Ga, USA, December 2010.

[19] Y. Liu, J. Slotine, and A. Barabási, "Controllability of complex networks," Nature, vol. 473, no. 7346, pp. 167-173, 2011.

[20] C. Godsil and G. Royle, Algebraic Graph Theory, vol. 207, Springer, New York, NY, USA, 2001. 


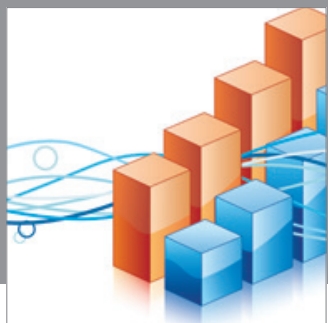

Advances in

Operations Research

mansans

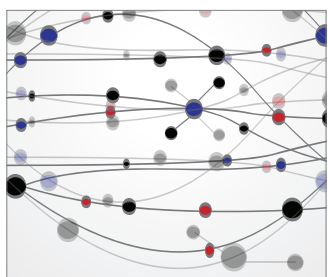

The Scientific World Journal
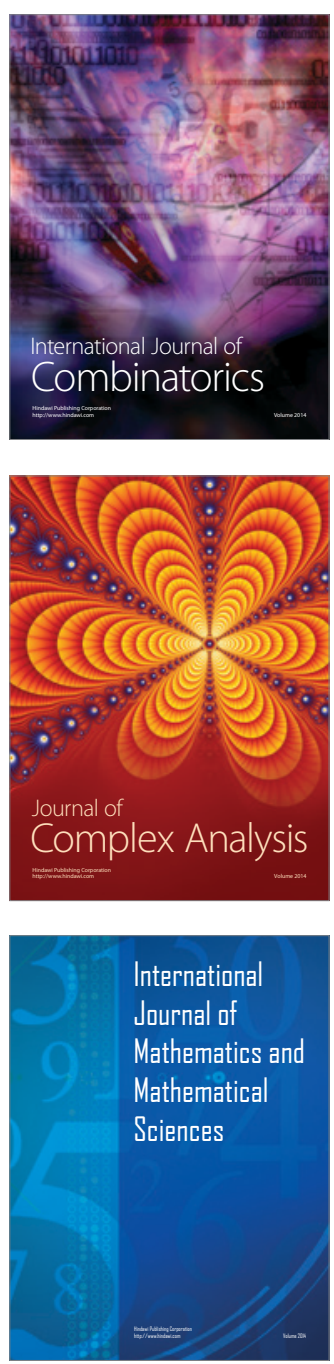
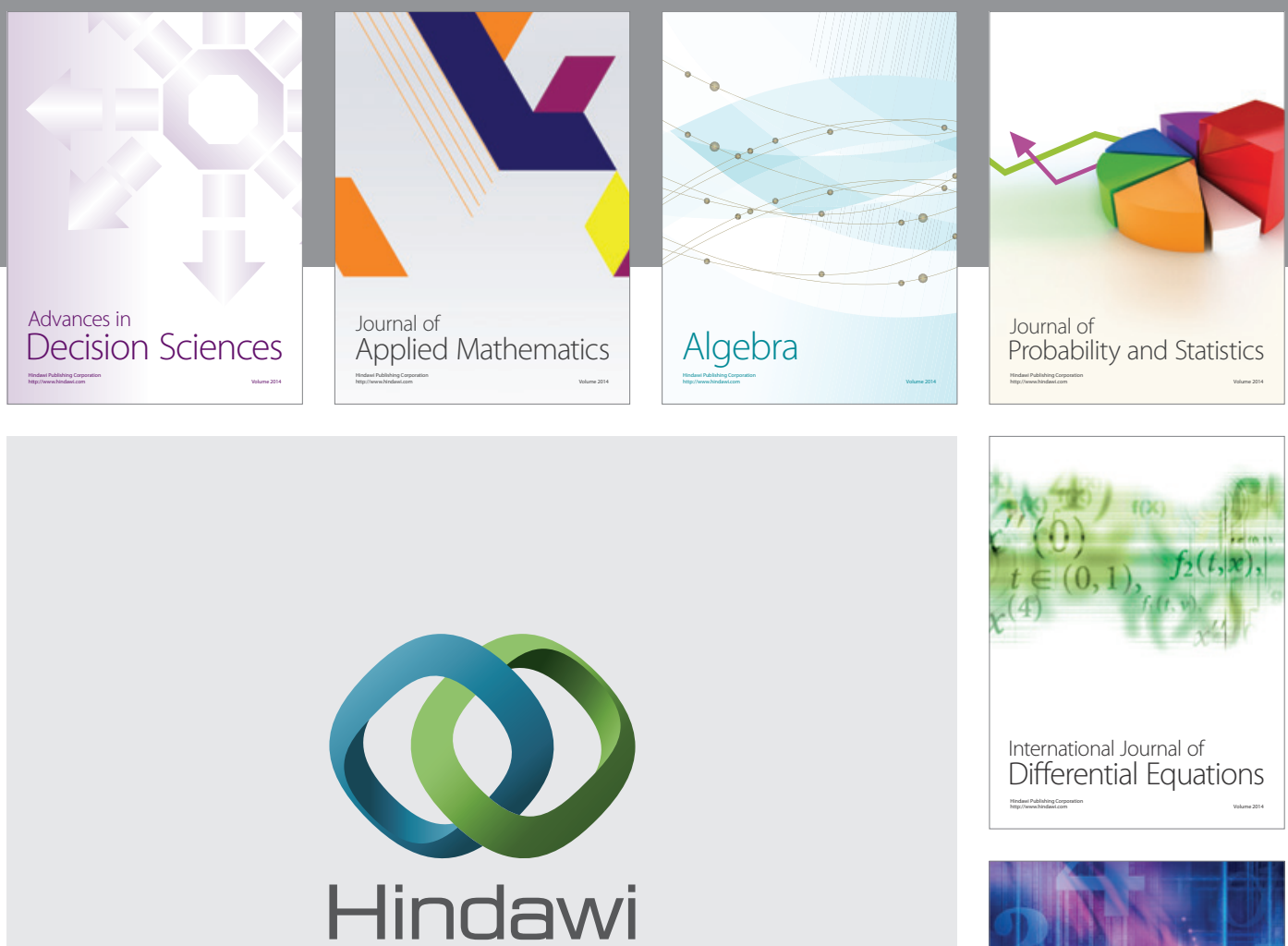

Submit your manuscripts at http://www.hindawi.com
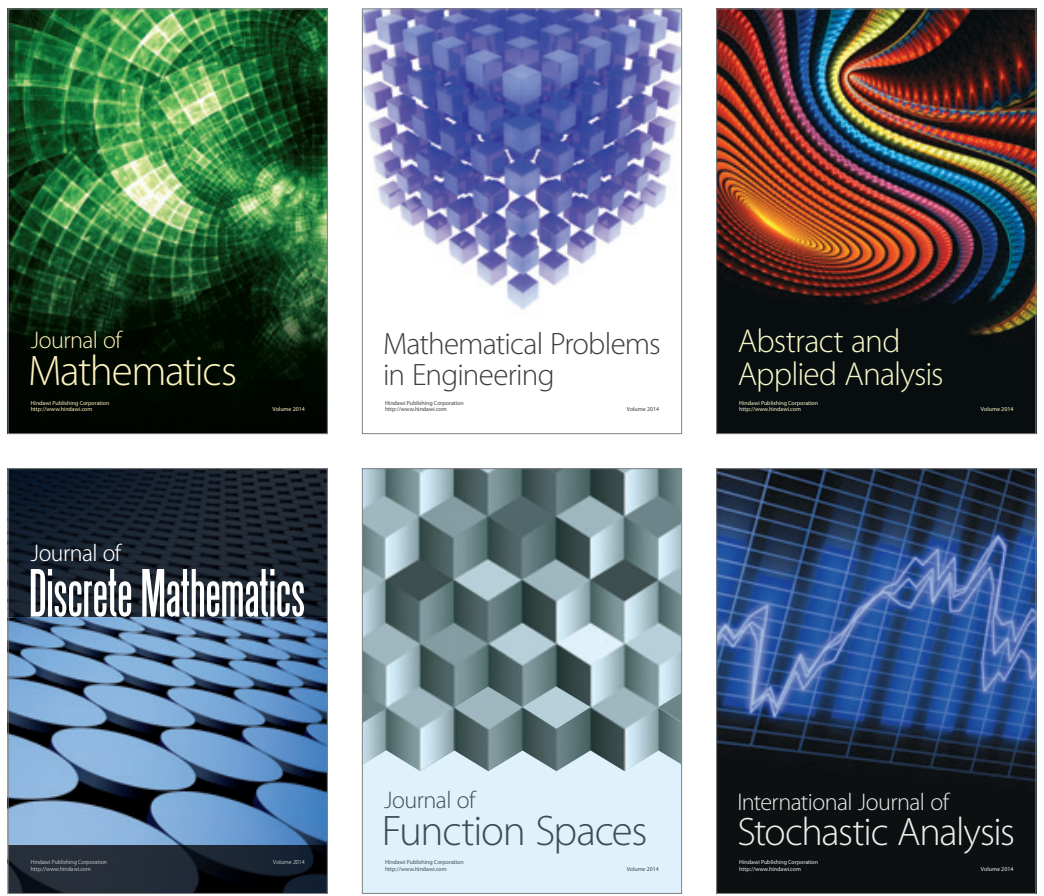

Journal of

Function Spaces

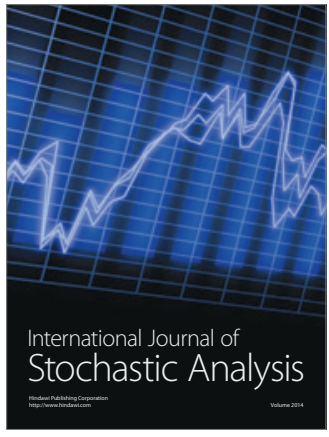

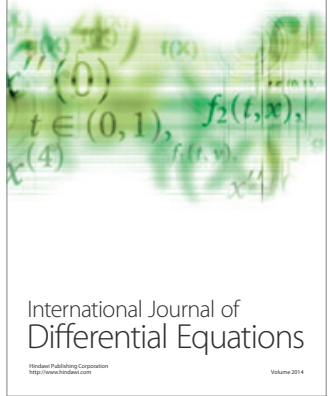
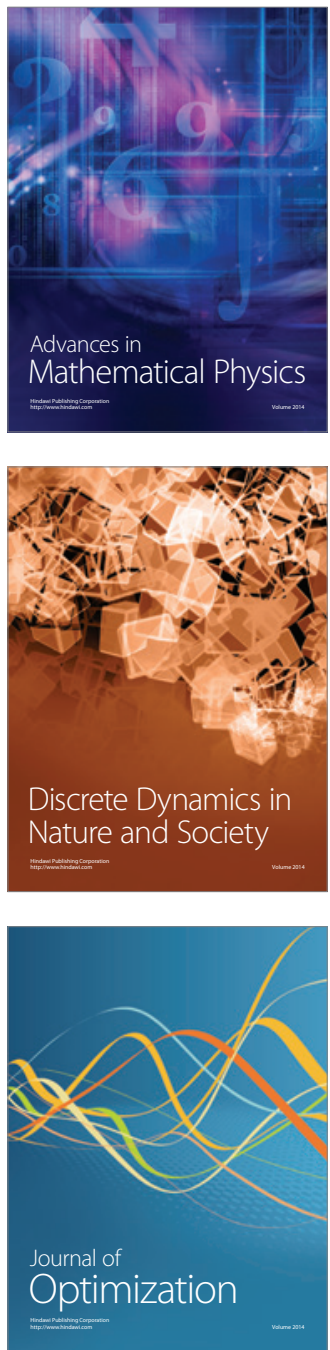